\title{
Stochastic Geometry Analysis for Band-Limited Terahertz Band Communications
}

\author{
Joonas Kokkoniemi, Janne Lehtomäki, and Markku Juntti \\ Centre for Wireless Communications (CWC), \\ University of Oulu, P.O. Box 4500, 90014 Oulu, Finland \\ Email: joonas.kokkoniemi@oulu.fi
}

\begin{abstract}
The terahertz band (0.1-10 $\mathrm{THz})$ point-to-point links offer very large spectral resources for extremely high data rate links or an ability to share the resources among large numbers of users or devices. The latter case causes a need to consider interference in the $\mathrm{THz}$ band networks. The stochastic geometry is a powerful tool to estimate the network level interference and its moments in the case of random networks. This paper extends the previous works by considering bandwidth limited networks, i.e., instead of generic modeling of the interference, we apply bandwidth limited transmissions to better model realistic networks that utilize limited resources. Furthermore, we assume heterogenous network with some of the nodes utilizing directional antennas, and others using isotropic antennas. Network is further assumed to be sparse in a sense that the noise causes partial limitations in the achievable signal-tonoise-plus-interference (SINR). The mean interference and the SINR are derived and their validity is verified with computer simulations.
\end{abstract}

\section{INTRODUCTION}

The millimeter wave $(30-300 \mathrm{GHz})$ and terahertz band $(0.1-10 \mathrm{THz})$ communication technologies have become very popular research topics during the past few year in the search of extremely high data rates for future communication systems. Especially, the $\mathrm{THz}$ band can be seen as a very potential platform to realize one Tbps links due to the vast available frequency resources. On the other hand, the high available bandwidth makes it possible to share the frequency resources among large numbers of users and devices. Large bandwidth can also simplify medium access control (MAC) design for resource limited devices. The latter is of interest to future communications systems as the number of internet of things (IoT) devices is expected increase strongly [1], and thus, they play an important part in the considered applications for these high frequency bands.

The downside of the otherwise very potential frequency bands is the extremely high path loss and molecular absorption loss [2], especially in the $\mathrm{THz}$ band, the main interest of this paper. Those limit the maximum operational distances, but on the other hand, high loss allows communication in the short range without causing interference to the distant devices. A second downside is the lack of technologies to efficiently utilize these frequencies. One of the most promising materials in developing novel technologies for future transceivers is graphene, that is suitable for very high efficiency components for high frequency applications, such as antennas [3]. Graphene, as well as other promising materials and the general development of the high frequency components bring confidence to the future of the $\mathrm{THz}$ band. A problem with $\mathrm{THz}$ frequencies has been that sufficient output power is difficult to achieve. Current developments on $\mathrm{THz}$ are focused on generating high power signals at the $300 \mathrm{GHz}$ frequency band, but potential solutions exist for even higher frequencies.

The $\mathrm{THz}$ band point-to-point links will be the reality in the future. This is only one step away from the $\mathrm{THz}$ band networks. We know the properties of a line-of-sight (LOS) channels in the $\mathrm{THz}$ band. However, the collective network behavior is more difficult to predict, especially when considering that the high frequency links will utilize directional antennas as a consequence of the high path loss. We can utilize simulation models to predict the interference in the network, but there is also a better tool; stochastic geometry. It is a very powerful tool in estimation of the interference and its moments in random networks. It relies on geometrical properties of the environment and node distributions and the expected values of the randomly distributed distances and node orientations. This paper is an extension to our previous papers on the THz band stochastic geometry [4], [5]. Those modeled generic $\mathrm{THz}$ band communications considering deterministic molecular absorption loss and a full free space path loss model that is commonly used in the THz band channel modeling. In those papers, we assumed interference limited network and generic per-Hertz behavior of the channel. This paper extends those to consider band-limited transmission and smaller user densities in order to look into the noise and interference limited signal-to-noise-plus-interference (SINR) cases. This is strongly frequency dependent as the path loss changes a lot over the entire $\mathrm{THz}$ band. We also utilize less generic representation of the system geometry by restricting the node distribution to be on a plane with three dimensional path loss in order to have more realistic representation of a network. Results significantly differ from those in our earlier papers and show different trends due to results here represent average behavior of the interference over a certain band. Compared to earlier works, this work led to new insights on interference in $\mathrm{THz}$ networks, since the strongly frequency dependent molecular absorption loss flattens as it is averaged over a frequency band and offers a clearer view for the actual network behavior.

There are numerous papers on stochastic geometry for estimating the interference in wireless networks, such as [6]- 
[11]. Our model follows those rather accurately, but with the differences coming from utilization of the molecular absorption and strict LOS assumption. Those cause some problems, but closed-form solutions of the moments of the interference can be derived.

There are some works on stochastic geometry for the $\mathrm{THz}$ band networks [12]-[14]. Those works have one major difference to this paper, as well as to our previous papers on the stochastic geometry for $\mathrm{THz}$ band networks [4], [5]. They assume the Matern's hard-core process, which utilizes guard bands around the Txs and Rxs. This is a dependent thinning of a Poisson distributed network and is very useful in scenarios where node locations can be controlled. However, our approach is more general and is therefore more easy to extend into various scenarios.

The rest of this paper is organized as follows. Section II introduces the system model. Section III gives the channel model, antenna assumptions, and derives the moments of the interference. Finally, numerical results/examples are given in Section IV and Section V concludes the paper.

\section{SySTEM MOdEL}

The system model herein is closely following the one we presented in [5]. That is, we consider $K$ different node categories that can have their unique transmit powers, antenna configurations, transmit probabilities, and node densities. The node distributions are assumed to follow the Poisson distribution in all the $K$ categories, or network layers. In the numerical results we utilize two node categories, the isotropic sensor nodes and the directional gateway nodes. Such a network configuration is illustrated in Fig. 1. It depicts a network where simple sensor nodes utilize isotropic antenna and more complex gateways are capable of beam steering and can direct their transmissions towards the desired receiver.

The core idea of the stochastic geometry is to model a random network with random node locations to characterize the average behavior of the network. Due to ease of calculations, the interference is usually studied about a node at the origin and the transmitting nodes are dropped around it (like in Fig. 1). The node at the origin is therefore called a typical node, as it sees the typical interference behavior of the network. Each randomly dropped node is associated with its receiver in random direction from the transmitter. Thus, the typical node in the origin sees the network as a random interferer. Placing the typical node in the origin helps with the calculations, since we assume circularly symmetric planar drop area and therefore the interference geometry of the system is obtained as a function of the distance from the typical node only. This is due to the dropped nodes are modeled as densities rather than actual nodes, and thus, the aggregate interference is obtained by integrating over the space surrounding the typical node. More information of the assumptions and properties of this type of a network can be found, e.g., in our paper about the stochastic geometry for the $\mathrm{THz}$ band [4] and in the literature for the general stochastic geometry [6]-[11].

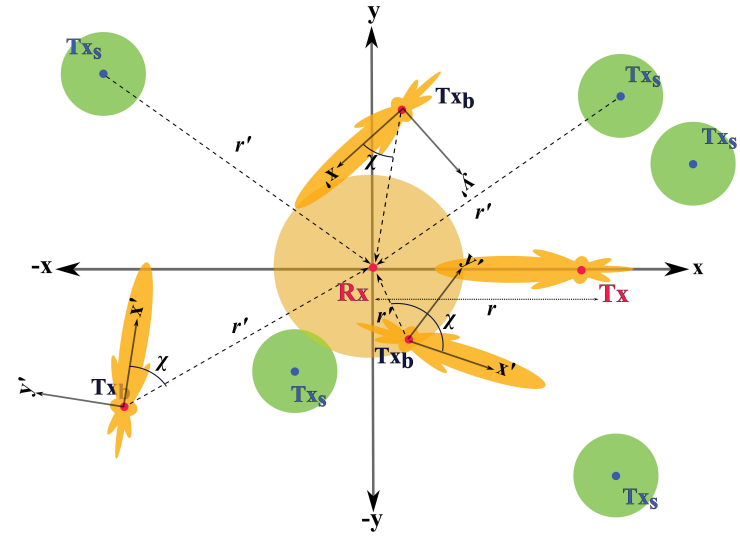

Fig. 1. The system model. The typical node at the origin corresponds to a random point in space that experiences the interference induced by randomly distributed nodes around it. All the nodes, including the typical node, can have either directional or isotropic antennas.

\section{Channel model And Stochastic Geometry For THE THZ BAND}

\section{A. Path Loss Model}

In our previous works we assumed fully general path loss model that is valid in all possible spatial dimensions [4], [5]. Here, on the other hand, we assume regular three dimensional path loss and a planar drop area for the nodes as detailed above due to interest in more realistic network deployment scenario. The path loss model herein is a LOS model that takes into account the free space path loss (FSPL) and molecular absorption loss [2]. Then the path gain of the channel is given by

$$
l(r, f)=\frac{c^{2} \exp \left(-\kappa_{\mathrm{a}}(f) r\right)}{(4 \pi r f)^{2}},
$$

where $\kappa_{\mathrm{a}}(f)$ is the absorption coefficient at frequency $f, r$ is the distance from Tx to Rx, and $c$ is the speed of light. The absorption coefficient can be calculated with the help of databases, such as the HITRAN database [15], and as presented, e.g., in [2]. The main benefit of utilizing generic FSPL model is the ease of extending it to take into account additional propagation mechanisms (such as the molecular absorption here).

\section{B. Interference and Noise}

We are interested in the mean interference of band-limited networks, i.e., each node utilized certain common-to-all bandwidth $W$ at the same center frequency. Then the mean interference needs to be calculated over that bandwidth $W$. Given the above path loss model, the aggregate interference over that band becomes

$$
I_{\text {aggr }}=\sum_{k=1}^{K} \sum_{i \in \Phi_{k}} l_{k}\left(r_{i}\right)
$$

where

$$
l_{k}\left(r_{i}\right)=\int_{W} \frac{P_{\mathrm{Tx}, \mathrm{k}}}{W} \mathbb{E}_{\Theta}\left[G_{\mathrm{T} x, \mathrm{k}}(\Theta)\right] \mathbb{E}_{\Theta}\left[G_{\mathrm{Rx}, \mathrm{k}}(\Theta)\right] l\left(r_{i}, f\right) d f .
$$


where $\Phi_{k}$ is the set of interfering nodes, $P_{\mathrm{Tx}, \mathrm{k}}$ is the total transmit power of the $k$ th category Txs, $P_{\mathrm{Tx}, \mathrm{k}} / W$ is the per Hertz transmit power (we assume equal distribution of transmit power over the band $W), \mathbb{E}_{\Theta}\left[G_{\mathrm{Tx}, \mathrm{k}}(\Theta)\right]$ and $\mathbb{E}_{\Theta}\left[G_{\mathrm{Rx}, \mathrm{k}}(\Theta)\right]$ are the expected antenna gains of the Txs and the typical Rx node (assumed to be independent of the frequency), and $\Theta$ is the direction of the antenna in 3D space. The transmit power spectral density (PSD) $P_{\mathrm{Tx}, \mathrm{k}} / W$ can replaced with any arbitrary and application specific transmit PSD. The summation over $k$ node categories is done based on the previously mentioned assumption of the Poisson distributed nodes, and the fact that superimposing multiple PPPs is still a PPP [10, Sec. 1.3].

Based on the aggregate interference and path loss for the desired signal, signal-to-interference-plus-noise ratio (SINR) becomes

$$
S I N R=\frac{S(r)}{N+I_{\text {aggr }}},
$$

where

$$
S(r)=\int_{W} \frac{P_{\mathrm{Tx}}}{W} \max _{\Theta}\left[G_{\mathrm{Tx}}(\Theta)\right] \max _{\Theta}\left[G_{\mathrm{Rx}}(\Theta)\right] l(r, f) d f
$$

is the desired signal power, where $\max _{\Theta}[G]$ is the maximum antenna gain, assuming that the desired link is perfectly aligned. The noise power $N$ given by

$$
N=\int_{W} k_{b} T N_{f} n_{f}(f) d f
$$

where $k_{b}$ is the Boltzmann constant, $T$ is the temperature, $N_{f}$ is the noise figure, and $n_{f}$ is a noise reduction factor due to quantum effects at high frequencies [16], which is given by

$$
n_{f}(f)=\frac{h f}{k_{b} T}\left(\exp \left(\frac{h f}{k_{b} T}\right)-1\right)^{-1}
$$

where $h$ is the Planck's constant. This factor causes a few $\mathrm{dB}$ drop of the noise power at higher end of the $\mathrm{THz}$ band as a consequence of the high energy transitions between the energy states of the molecules becoming sparser when compared to the lower frequencies.

\section{Antenna Patterns}

The antenna patterns utilized here are either isotropic or simple cone shaped directional antenna patterns [4]. We always assume that the antenna pattern integrates to unity, and thus, the total transmit power over the antenna pattern is always constant. Therefore, the expected antenna gain to any given random direction $\Theta$ becomes

$$
\mathbb{E}_{\Theta}[G(\Theta)]=\frac{1}{4 \pi} \int_{\mathbb{R}^{3}} G(\Theta) d \mathbb{R}^{3}=\frac{1}{4 \pi},
$$

where $1 / 4 \pi$ is the effective expected antenna gain due to the above mentioned integration of the antenna pattern to unity. As a consequence, integrating $1 / 4 \pi$ over the $3 \mathrm{D}$ space equals unit total transmit power in average per unit transmit power.

\section{Stochastic Model of the Aggregate Interference}

The most interesting part of the stochastic modeling of the interference is the mean interference power that can be utilized in estimation of the expected interference of a network. The moments of the interference can be calculated from the Laplace transform of the aggregate interference [6], [7]

$$
\mathcal{L}_{I_{\text {aggr }}}(s)=\mathbb{E}_{\Phi}\left[\exp \left(-s \sum_{k=1}^{K} \sum_{i \in \Phi_{k}} l_{k}\left(r_{i}\right)\right)\right]
$$

where $\Phi$ indicates expectation over all the sets of nodes $\Phi_{k}$. The detailed calculations of this expression to the point from which we can calculate the moments of the interference are given in [4], [5]. One needs to add the above integration over a band $W$ and the associated terms herein to take into account the impact of calculating the interference over potentially large bandwidth in which the channel response may change drastically due to, e.g., molecular absorption loss. After some manipulations, we can give the Laplace transform as

$$
\mathcal{L}_{I_{\text {aggr }}}(s)=\prod_{K} \exp \left[-2 \pi p_{k} \lambda_{k} \int_{0}^{\infty} r\left(1-\exp \left(-s l_{k}(r)\right)\right) d r\right],
$$

where $\lambda_{k}$ is the density of the nodes of the node category $k$ and $p_{k}$ is the probability of a node to transmit, which operates as an effective thinning operation for the $\lambda_{k}$ due to assumption of an ALOHA transmission scheme.

From the above expression we can calculate the moments of the interference. The $n$th raw moment of the aggregate interference power is obtained from the $n$th derivative of the Laplace transform by [9]

$$
\mathbb{E}\left[I^{n}\right]=\left.(-1)^{n} \frac{d^{n}}{d s^{n}} \mathcal{L}_{I_{\text {aggr }}}(s)\right|_{s=0} .
$$

To give the final moments more easily, we mark the above Laplace transform in (10) as

$$
\mathcal{L}_{I_{\text {aggr }}}(s)=f_{G}(n) \exp (-L(s)),
$$

where

$$
f_{G}(n)=\left(\frac{\max _{\Theta}\left[G_{\mathrm{Tx}, \mathrm{k}}(\Theta)\right] \max _{\Theta}\left[G_{\mathrm{Rx}, \mathrm{k}}(\Theta)\right]}{\mathbb{E}_{\Theta}\left[G_{\mathrm{Tx}, \mathrm{k}}(\Theta)\right] \mathbb{E}_{\Theta}\left[G_{\mathrm{Rx}, \mathrm{k}}(\Theta)\right]}\right)^{n-1},
$$

where $n$ is the derivative order, is an antenna gain factor that takes into account the larger variability of the received power in the case of directional antennas because the maximum interference power varies between zero and $P_{\mathrm{Tx}, \mathrm{k}} \max _{\Theta}\left[G_{\mathrm{Tx}, \mathrm{k}}(\Theta)\right] \max _{\Theta}\left[G_{\mathrm{Rx}, \mathrm{k}}(\Theta)\right]$ [5]. It can be seen that this factor is one for isotropic antennas and does not have impact on the mean interference, as the expected antenna gains alone take into account the antenna effects for the average interference. The term $L(s)$ in Eq. (12) is

$$
L(s)=\sum_{K} 2 \pi p_{k} \lambda_{k} \int_{0}^{\infty} r\left(1-\exp \left(-s l_{k}(r)\right)\right) d r .
$$


Calculating the moments of the interference according to (11), first two moments become:

$$
\begin{gathered}
\mathbb{E}\left[I_{\text {aggr }}\right]=L^{\prime}, \\
\mathbb{E}\left[I_{\text {aggr }}^{2}\right]=-f_{G}(n=2) L^{\prime \prime}+\left(L^{\prime}\right)^{2},
\end{gathered}
$$

where

$$
\begin{aligned}
L^{\prime}(s=0) & =\sum_{K} \frac{c^{2}}{8 \pi} p_{k} \lambda_{k} \mathbb{E}_{\Theta}\left[G_{\mathrm{Tx}, \mathrm{k}}(\Theta)\right] \mathbb{E}_{\Theta}\left[G_{\mathrm{Rx}, \mathrm{k}}(\Theta)\right] \\
& \times \int_{0}^{\infty} r^{-1} \int_{W} \frac{P_{\mathrm{Tx}, \mathrm{k}}}{W f^{2}} \exp \left(-\kappa_{\mathrm{a}}(f) r\right) d f d r \\
L^{\prime \prime}(s=0) & = \\
& -\sum_{K} \frac{c^{4}}{128 \pi^{3}} p_{k} \lambda_{k}\left(\mathbb{E}_{\Theta}\left[G_{\mathrm{Tx}, \mathrm{k}}(\Theta)\right] \mathbb{E}_{\Theta}\left[G_{\mathrm{Rx}, \mathrm{k}}(\Theta)\right]\right)^{2} \\
& \times \int_{0}^{\infty} r^{-3} \int_{W} \frac{P_{\mathrm{Tx}, \mathrm{k}}^{2}}{W^{2} f^{4}} \exp \left(-2 \kappa_{\mathrm{a}}(f) r\right) d f d r .
\end{aligned}
$$

The mean interference is obtained directly from (15) and the variance is given by

$$
\operatorname{var}\left(I_{\text {aggr }}\right)=-f_{G}(n=2) L^{\prime \prime}(s=0),
$$

because $\operatorname{var}(X)=\mathbb{E}\left[X^{2}\right]-(\mathbb{E}[X])^{2}$, which is easy to solve from (15) and (16). Any higher moment can be calculated similarly and as shown for the third moment in [5].

\section{Numerical Results}

The numerical results are given for two layered network; one utilizing directional antennas and one utilizing isotropic antennas. The aggregate interference this case is a summation over the two node categories, but results are given for single layers as well in order to show the differences in the results as a consequence of different parameters. Those are given in Table I. The densities of the nodes are obtained from the drop area of four meters in diameter. This diameter is enough to ensure no interference coming outside this area, and thus, the simulations results approach to those obtained with theories presented in the previous section utilizing infinite area with the given node densities. The feasibility of the four meter diameter drop area is therefore shown by the perfect match between the theories and simulations. The simulation model for this paper is a band-limited modification to that given in detail in [4]. Furthermore, the bandwidth is assumed to be 20 Gigahertz, which offers very large theoretical capacity, but on the other hand it reduces the communication distance due to large noise power as it will be seen below. The noise figure $N_{f}$ of the receiver was assumed to be $10 \mathrm{~dB}$.

Using the above parameters, and calculated the mean interferences for both layers, their sum, desired signal powers at $11 \mathrm{~cm}$ distance from directional and isotropic transmitters, and the noise floor are given in Fig. 2 for the $20 \mathrm{GHz}$ bandwidth as a function of the center frequency. Fig. 3 is a closeup of Fig. 2 for the below one $\mathrm{THz}$ frequencies. The ripple in the curves
TABLE I

PARAMETERS FOR THE NUMERICAL RESULTS.

\begin{tabular}{|c|c|c|}
\hline \hline Parameter & Directional nodes & Isotropic nodes \\
\hline Antenna beam width & $\pi / 4$ & N/A \\
Number of nodes & 100 & 300 \\
Density of nodes $\lambda_{k}$ & $2 / \mathrm{m}^{2}$ & $6 / \mathrm{m}^{2}$ \\
Transmit power $P_{\mathrm{Tx}, \mathrm{k}}$ & $1 \mathrm{~W}$ & $1 \mathrm{~W}$ \\
Probability to transmit $p_{k}$ & 0.5 & 1 \\
\hline
\end{tabular}

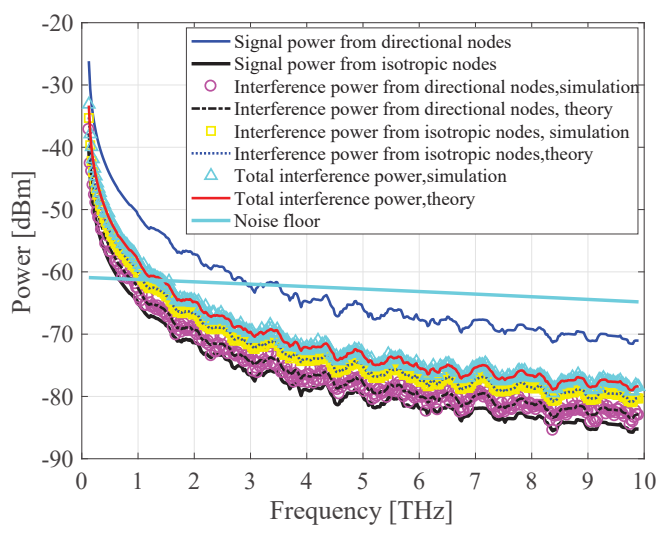

Fig. 2. The aggregate interferences, signal powers, and noise floor based on the theory and simulations.



Fig. 3. A closeup of Fig. 2 for below one THz frequencies.

is caused by the molecular absorption that causes frequency selective, but deterministic fading to the signals. Therefore, it gives similar fading to all the frequency domain powers. We can see that the high bandwidth combined with the large path loss results in interference limited communications below about one $\mathrm{THz}$ and noise limited above it. Obviously the noise limitations is subject to the bandwidth of the transmission. The communication distance here is very low and could be enhanced with higher gain antennas. However, the point here is not to demonstrate a system performance, but the performance of the theories for the band-limited interference. As it can be seen, the theories presented in the previous section perfectly match with those obtained by computer simulations.

Fig. 4 gives the corresponding SINR values to the powers given in Fig. 2. Fig. 5 is a closeup of Fig. 4 for the below one $\mathrm{THz}$ frequencies. As it can be expected, the theoretical SINRs based on the stochastic interferences perfectly matches 


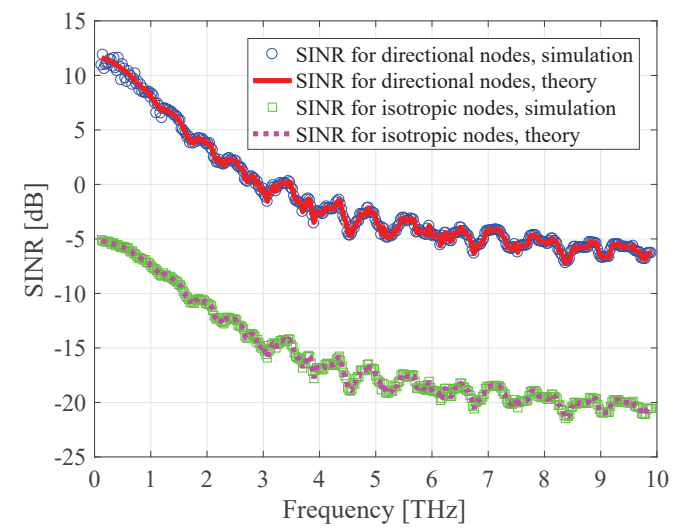

Fig. 4. SINRs for the directional and isotropic nodes with interference coming from the total aggregate interference power of the network.



Fig. 5. A closeup of Fig. 2 for below one THz frequencies.

the simulation results. Although not the point of these results, the SINRs show the importance of the directional antennas in the THz band communications: in the case of rather dense network of nodes, interference and noise quickly cover the weak transmit power of an isotropic transmitter. Therefore, the $\mathrm{THz}$ band communications will always require directional antennas, even at very low distance, but even more so if we target several meters of range. In the case of larger link distances, lower bandwidths should be utilized due to quickly aggregating noise power as a function of the bandwidth. Below one $\mathrm{THz}$ frequency and especially in the millimeter wave frequencies the stochastic interference plays very important role due to relatively lower path loss compared to the above one $\mathrm{THz}$ frequencies.

These results show that the stochastic geometry is a useful tool to estimate the band-limited systems' interference levels. Although, the noise might be more meaningful in many cases, the accurate link budget calculations require full understanding of the energies in the channel. Those can be estimated quickly and accurately with the stochastic geometry without a need for complex simulation models.

\section{CONCLUSion}

We have show the aggregate interference model for bandlimited networks operating at the $\mathrm{THz}$ frequency band. Based on the simulations, these models predict the interference levels exactly as well as the higher moments of the interference. With the help of stochastic geometry, the moments can be obtained without the need for heavy network level simulations, but the interference can be calculated with closed form expressions. Based on the knowledge of the interference in the network, accurate link budget calculations become possible by adjusting the modulations, transmit powers, etc. to the expected SINR of an individual link. This is a very important aspect on which the stochastic geometry can help a lot by accelerating the estimation process without sacrificing the accuracy in the process.

\section{ACKNOWLEDGEMENT}

This project (TERRANOVA) has received funding from Horizon 2020, European Union's Framework Programme for Research and Innovation, under grant agreement No. 761794.

\section{REFERENCES}

[1] G. P. Fettweis, "5G and the future of IoT," in Proc. European Solid-State Circuits Conf., 2016, pp. 21-24.

[2] J. M. Jornet and I. F. Akyildiz, "Channel modeling and capacity analysis for electromagnetic nanonetworks in the terahertz band," IEEE Trans. Wireless Commun., vol. 10, no. 10, pp. 3211-3221, Oct. 2011.

[3] — " "Graphene-based plasmonic nano-antenna for terahertz band communication in nanonetworks," IEEE J. Sel. Areas Commun., vol. 31, no. 12, pp. 685-694, Dec. 2013.

[4] J. Kokkoniemi, J. Lehtomäki, and M. Juntti, "Stochastic geometry analysis for mean interference power and outage probability in thz networks," IEEE Trans. Wireless Commun., vol. 16, no. 5, pp. $3017-$ 3028, May 2017.

[5] — "Stochastic analysis of multi-tier nanonetworks in thz band," in Proc. ACM Int. Conf. Nanoscale Comput. Commun., 2017, pp. 1-6.

[6] M. Haenggi and R. K. Ganti, "Interference in large wireless networks," Foundations and Trends in Networking, vol. 3, no. 2, pp. 127-248, Nov. 2008.

[7] M. Haenggi, J. G. Andrews, F. Baccelli, O. Dousse, and M. Franceschetti, "Stochastic geometry and random graphs for the analysis and design of wireless networks," IEEE J. Sel. Areas Commun., vol. 27, no. 7, pp. 1029-1046, Sep. 2009.

[8] M. Haenggi, "Outage, local throughput, and capacity of random wireless networks," IEEE Trans. Wireless Commun., vol. 8, no. 8, pp. 4350-4359, Aug. 2009.

[9] H. ElSawy, E. Hossain, and M. Haenggi, "Stochastic geometry for modeling, analysis, and design of multi-tier and cognitive cellular wireless networks: A survey," IEEE Commun. Surveys Tuts., vol. 15, no. 3, pp. 996-1019, Jun. 2013.

[10] F. Baccelli and B. Blaszczyszyn, "Stochastic geometry and wireless networks, volume I - Theory," Foundations and Trends in Networking, vol. 3, no. 3-4, pp. 249-449, Dec. 2009.

[11] A. Baddeley, Stochastic Geometry: Spatial Point Processes and their Applications, ser. Lecture Notes in Mathematics, W. Weil, Ed. Springer Berlin Heidelberg, 2007.

[12] V. Petrov, D. Moltchanov, and Y. Koucheryavy, "Interference and SINR in dense terahertz networks," in Proc. IEEE Veh. Technol. Conf. (fall), 2015, pp. 1-5.

[13] _ - "On the efficiency of spatial channel reuse in ultra-dense $\mathrm{THz}$ networks," in Proc. IEEE Global Commun. Conf., 2015, pp. 1-7.

[14] V. Petrov, M. Komarov, D. Moltchanov, J. M. Jornet, and Y. Koucheryavy, "Interference and sinr in millimeter wave and terahertz communication systems with blocking and directional antennas," IEEE Trans. Wireless Commun., vol. 16, no. 3, pp. 1791-1808, Mar. 2017.

[15] L. S. Rothman et al., "The HITRAN 2012 molecular spectroscopic database," J. Quant. Spectrosc. Radiat. Transfer, vol. 130, no. 1, pp. 4-50, Nov. 2013.

[16] H. Nyquist, "Thermal agitation of electric charge in conductors," Phys. Rev., vol. 32, pp. 110-113, July 1928. 